\title{
AN ALGEBRAIC PROPERTY OF THE ČECH COHOMOLOGY GROUPS WHICH PREVENTS LOCAL CONNECTIVITY AND MOVABILITY(1)
}

BY

\author{
JAMES KEESLING
}

ABSTRACT. Let $C$ denote the category of compact Hausdorff spaces and $H$ : $C \rightarrow H C$ be the homotopy functor. Let $S: C \rightarrow S C$ be the functor of shape in the sense of Holsztyński for the projection functor $H$. Let $X$ be a continuum and $H^{n}(X)$ denote $n$-dimensional Čech cohomology with integer coefficients. Let $A_{x}=\operatorname{char} H^{1}(X)$ be the character group of $H^{1}(X)$ considering $H^{1}(X)$ as a discrete group. In this paper it is shown that there is a shape morphism $F \in$ $\operatorname{Mor}_{S C}\left(X, A_{X}\right)$ such that $F^{*}: H^{1}\left(A_{X}\right) \rightarrow H^{1}(X)$ is an isomorphism. It follows from the results of a previous paper by the author that there is a continuous mapping $f: X \rightarrow A_{X}$ such that $S(f)=F$ and thus that $f^{*}: H^{1}\left(A_{X}\right) \rightarrow H^{1}(X)$ is an isomorphism. This result is applied to show that if $X$ is locally connected, then $H^{1}(X)$ has property L. Examples are given to show that $X$ may be locally connected and $H^{n}(X)$ not have property $\mathrm{L}$ for $n>1$. The result is also applied to compact connected topological groups.

In the last section of the paper it is shown that if $X$ is compact and movable, then for every integer $n, H^{n}(X) /$ Tor $H^{n}(X)$ has property $\mathrm{L}$. This result allows us to construct peano continua which are nonmovable. An example is given to show that $H^{n}(X)$ itself may not have property $\mathrm{L}$ even if $X$ is a finitedimensional movable continuum.

Introduction. Let $C$ denote the category of compact Hausdorff spaces and $H: C \rightarrow H C$ be the homotopy functor. Let $S: C \rightarrow S C$ be the functor of shape in the sense of Holsztynski [3] for the projection functor $H$. Let $X$ be a continuum and let $H^{n}(X)$ denote $n$-dimensional Čech cohomology with integer coefficients. Let $A_{X}$ be the character group of $H^{1}(X)$ considering $H^{1}(X)$ as a discrete group. Note that $H^{1}(X)$ is torsion free and thus $A_{X}$ is a compact connected abelian topological group. In this paper we show that if $B$ is a compact connected abelian topological group and $b: H^{1}(B) \rightarrow H^{1}(X)$ is a homomorphism, then there is a shape morphism $F \in \operatorname{Mor}_{S C}(X, B)$ such that $F^{*}=b$. By the results in [6], there is a continuous function $f: X \rightarrow B$ with $S(f)=F$ and thus with $f^{*}=b$

Received by the editors November 28, 1972.

AMS (MOS) subject classifications (1970). Primary 55D99; Secondary 55B05, 22 B99.

Key words and phrases. Shape functor, Čech cohomology, compact space, compact connected topological group, local connectivity, movability, property $\mathbf{L}$, continuous homomorphism.

(1) This research was supported by NSF Grant GP-24616A\#1. 
also. In particular, there is an $f: X \rightarrow A_{X}$ such that $f^{*}: H^{1}\left(A_{X}\right) \rightarrow H^{1}(X)$ is an isomorphism. If $g: X \rightarrow B$ is any continuous map, then there 15 a continuous homomorphism $\pi: A_{X} \rightarrow B$ such that $\pi$ of is homotopic to $g$.

In the second section of the paper we apply the results of the first section to show that if $X$ is a locally connected continuum, then $H^{1}(X)$ must have property $L$. We construct examples of peano continua such that $H^{n}(X)$ is torsion free and does not have property $L$ for $n>1$. This construction is used to construct the nonmovable peano continua in the last section of the paper.

In the third section of the paper, it is shown that, for $G$ a compact connected topological group and $D$ the commutator subgroup of $G$, the map $f: G \rightarrow G / D$ induces an isomorphism $f^{*}: H^{1}(G, D) \rightarrow H^{1}(G)$.

In the last section of the paper movability of compact spaces is studied. It is shown that if $X$ is compact and movable, then $H^{n}(X) /$ Tor $H^{n}(X)$ has property $\mathrm{L}$ for all $n$. This allows us to construct peano continua which are not movable. In particular we construct $2^{x_{0}}$ nonhomeomorphic nonmovable $n$-dimensional peano continua for each $n>1$. This generalizes a result of Borsuk [1]. This construction does not work for $n=1$, since a one-dimensional peano continuum is movable [9]. These results show that if $X$ is an $n$-dimensional $L C^{n-1}$ peano continuum, then $H^{k}(X) /$ Tor $H^{k}(X)$ has property $\mathrm{L}$ for all $k$.

An example is given to show that $H^{n}(X)$ may not have property $L$ even if $X$ is a finite-dimensional movable continuum.

Notation. We assume the notation of [5] and [6]. It is expected that the reader is familiar with the approaches to shape theory by Holsztyński [3] and Mardesič and Segal [8].

Some knowledge of topological groups is assumed as contained in [10]. If $X$ is a compact space, $H^{n}(X)$ denotes $n$-dimensional Čech cohomology with integer coefficients.

1. Homomorphisms of Čech cohomology groups which induce shape mor phisms. Let $B$ be a compact connected abelian topological group and $b: H^{1}(B) \rightarrow H^{1}(X)$ be a homomorphism where $X$ is a continuum. In this section of the paper it is shown that there is a unique shape morphism $F$ from $X$ to $B$ such that $F^{*}=b: H^{1}(B) \rightarrow H^{1}(X)$. Some immediate corollaries are derived in this section also. The next two sections of the paper then give further applications of these results. The basic theorem is the following.

1.1. Theorem. Let $X$ be a continuum and $B$ a compact connected abelian topological group. Suppose that $b: H^{1}(B) \rightarrow H^{1}(X)$ is a bomomorphism. Then there is a unique shape morpbism $F \in \operatorname{Mor}_{S C}(X, B)$ such that $F^{*}=b: H^{1}(B) \rightarrow H^{1}(X)$.

Before we can prove Theorem 1.1, we will need to quote some theorems. 
1.2. Theorem (Huber [4]). Let $X$ be compact. Then $H^{n}(X)$ is naturally isomorpbic to $\operatorname{Mor}_{H C}(X, K(Z, n))$.

In particular, $H^{1}(X)$ is naturally isomorphic to $\operatorname{Mor}_{H C}(X, T)$ where $T$ is the circle.

1.3. Theorem (Scheffer [11]). Let $G$ be a compact connected topological group and $A$ a compact connected abelian topological group. Then if $f: G \rightarrow A$ is continuous with $f(e)=0$, then $f$ is homotopic to a unique continuous bomomorphism.

The next theorem follows from Theorems 1.2 and 1.3 and is due to N. Steenrod.

1.4. Theorem (Steenrod [13]). Let $A$ be a compact connected abelian topological group. Then char $A$ is naturally isomorpbic to $H^{1}(A)$.

Proof. By Theorem 1.2, $H^{1}(A)$ is naturally isomorphic to $\operatorname{Mor}_{H C}(A, T)$. By Theorem 1.3, $\operatorname{Mor}_{H C}(A, T)$ is naturally isomorphic to $\operatorname{Hom}(A, T)=\operatorname{char} A$. Thus $H^{1}(A)$ is naturally isomorphic to char $A$.

Proof of Theorem 1.1. Using the natural isomorphism given by Theorems 1.2 and 1.4, we assume that $b$ is a homomorphism from char $B$ to $\operatorname{Mor}_{H C}(X, T)$. Now $B$ is the inverse limit of tori $\left\{T_{a}^{n a} ; \pi_{a \beta} ; a \leq \beta \in A\right\}$ where $\pi_{a \beta}\left(T_{\beta}^{n} \beta\right)=T_{a}^{n_{a}}$ is a continuous homomorphism. Let the projections making $B$ the inverse limit be $\pi_{a}(B)=T_{a}^{n a}$ for $a \in A$. We need to define the morphism $F \in \operatorname{Mor}_{S C}(X, B)$. We view the shape morphisms as functors from $H_{B}$ to $H_{X}$ as described in $\$ 4$ of [3]. To be a shape morphism $F$ must be the identity on the morphisms of $H_{B}$ to the morphisms of $H_{X}$. First we define $F$ on objects in $H_{B}$ of the form $H(\pi)$ where $\pi: B \rightarrow T^{n}$ is a continuous homomorphism. Then we will use the inverse system $\left\{T_{a}^{n_{a}}\right\}$ to extend the definition of $F$ to all of the objects of $H_{B}$.

Let $\pi: B \rightarrow T^{n}$ be a continuous homomorphism. Then let $T^{n}=T_{1} \times \cdots \times T_{n}$ be a factorization of $T^{n}$ into circle subgroups and let $e_{i}: T \rightarrow T^{n}$ and $p_{i}: T^{n} \stackrel{n}{\rightarrow}$ $T$ be the injection and projection homomorphisms for $i=1, \ldots, n$. Now $p_{i} \circ \pi \epsilon$ char $B$ and we let $f=\left(f_{1}, \cdots, f_{n}\right): X \rightarrow T^{n}=T_{1} \times \cdots \times T_{n}$ where $H\left(f_{i}\right)=b\left(p_{i} \circ \pi\right)$. Then define $F(H(\pi))=H(f)$. Since factorizations of $T^{n}$ are not unique, we need to show that $F(H(\pi))$ is well defined. So, let $T^{n}=T_{1}^{\prime} \times \cdots \times T_{n}^{\prime}$ be another factorization of $T^{n}$ into circle subgroups with injection homomorphisms $e_{i}^{\prime}: T \rightarrow$ $T^{n}$ and projection homomorphisms $p_{i}^{\prime}: T^{n} \rightarrow T$. Then there are integers $n_{i j}$ for $1 \leq i, j \leq n$ such that $\sum_{j=1}^{n} n_{i j} p_{j}^{\prime}=p_{i}$ for each $i$. Then if $1_{T^{n}}$ is the identity map on $T^{n}$, then $1_{T^{n}}: T_{1}^{0} \times \cdots \times T_{n}^{0} \rightarrow T_{1} \times \cdots \times T_{n}$ can be represented as $1_{T^{n}}=$ $\sum_{i=1}^{n} \sum_{j=1}^{n} e_{i} n_{i j} p_{j}^{\prime}$. Now let $f^{\prime}=\left(f_{1}^{\prime}, \cdots, f_{n}^{\prime}\right): X \rightarrow T_{1}^{\prime} \times \cdots \times T_{n}^{\prime}$ where $H\left(f_{i}^{\prime}\right)=$ $b\left(p_{i}^{\prime} \circ \pi\right)$. Since $b:$ char $B \rightarrow$ Mor $_{H C}(X, T)$ is a homomorphism, $b\left(p_{i} \circ \pi\right)=$ $b\left(\sum_{j=1}^{n} n_{i j} p_{j}^{\prime} \circ \pi\right)=\sum_{j=1}^{n} n_{i j} b\left(p_{j}^{\prime} \circ \pi\right)$. Thus $H\left(f_{i}\right)=\sum_{j=1}^{n} n_{i j} H\left(f_{j}^{\prime}\right)$. Therefore 
$\sum_{i=1}^{n} \sum_{j=1}^{n} n_{i j} H\left(f_{j}^{\prime}\right)=\sum_{i=1}^{n} H\left(f_{i}\right)=H(f)$. That is, $H\left(1_{T^{n}}\right) \circ H\left(f^{\prime}\right)=H(f)$. Thus $H(f)$ $=H\left(f^{\prime}\right)$ and $F(H(\pi))$ is well defined. Similarly one can verify that if $\pi: B \rightarrow T^{n}$ and $\pi^{\prime}: B \rightarrow T^{m}$ are continuous homomorphisms with $\pi^{\prime}=q \circ \pi$, then $F\left(H\left(\pi^{\prime}\right)\right)=$ $H(q) \circ F(H(\pi))$.

Now suppose that $p: B \rightarrow P$ is continuous with $P$ an $H$-object of $C$. Then let $a \in A$ and $p_{a}: T_{a}^{n_{a}} \rightarrow P$ be such that $H\left(p_{a}\right) \circ H\left(\pi_{a}\right)=H(p)$. Then define $F(H(p))=H\left(p_{\alpha}\right) \circ F\left(H\left(\pi_{a}\right)\right)$. We need to show that $F(H(p))$ is well defined. Suppose that $\beta \in A$ and $p_{\beta}: T_{\beta}^{n}{ }_{\beta} \rightarrow P$ with $H\left(p_{\beta}\right) \circ H\left(\pi_{\beta}\right)=H(p)$. Then let $\gamma \geq \alpha$ and $\beta$, and let $\delta \geq \gamma$ be such that $H\left(p_{\beta} \circ \pi_{\beta \gamma} \circ \pi_{\gamma \delta}\right)=H\left(p_{a} \circ \pi_{a \gamma} \circ \pi_{\gamma \delta}\right)$ using the fact that $H\left(p_{\beta} \circ \pi_{\beta \gamma} \circ \pi_{\gamma}\right)=H\left(p_{a} \circ \pi_{a \gamma} \circ \pi_{\gamma}\right)$. Then we have

$$
H\left(p_{a} \circ \pi_{a \delta}\right) \circ F\left(H\left(\pi_{\delta}\right)\right)=H\left(p_{a}\right) \circ F\left(H\left(\pi_{a}\right)\right)
$$

and

$$
H\left(p_{\beta} \circ \pi_{\beta \delta}\right) \circ F\left(H\left(\pi_{\delta}\right)\right)=H\left(p_{\beta}\right) \circ F\left(H\left(\pi_{\beta}\right)\right) .
$$

But since the left-hand sides of the above equalities are equal we have $H\left(p_{\alpha}\right) \circ F\left(H\left(\pi_{\alpha}\right)\right)=H\left(p_{\beta}\right) \circ F\left(H\left(\pi_{\beta}\right)\right)$ and $F(H(p))$ is well defined. We define $F(H(f))=H(f)$ for $H(f)$ a morphism in $H_{B}$ and then $F$ will be a shape morphism from $H_{B}$ to $H_{X}$.

Now we need to show that $F^{*}=b: \operatorname{char} B \rightarrow \operatorname{Mor}_{S C}(X, T)$. If $\chi \in \operatorname{char} A$, then $b(\chi)=F(H(\chi))$ by the way $F$ was defined. Thus $F^{*}=b$. Now if $F, G \in$ $\operatorname{Mor}_{S C}(X, B)$ with $F^{*}=G^{*}=h$, then for $\pi: B \rightarrow T^{n}$ a continuous homomorphism $F(H(\pi))=G(H(\pi))$ by repeating the first part of the proof for $G$. Thus, $F\left(H\left(\pi_{a}\right)\right)=$ $G\left(H\left(\pi_{\alpha}\right)\right)$ for all $\alpha \in A$ and thus $F=G$ by Lemma 4.1 of $[3, \mathrm{p} .162]$.

1.5. Corollary. Let $X$ be a continuum and $A_{X}=$ char $H^{1}(X)$. Then there is a shape morphism $F \in \operatorname{Mor}_{S C}\left(X, A_{X}\right)$ such that $F^{*}: H^{1}\left(A_{X}\right) \rightarrow H^{1}(X)$ is an isomorphism.

1.6. Corollary. Let $X$ be a continuum and $x \in X$. Let $B$ be a compact connected abelian topological group and let $b: H^{1}(B) \rightarrow H^{1}(X)$ be a homomorphism. Then there is a continuous map $f: X \rightarrow B$ with $f(x)=0$ such that $f^{*}=b: H^{1}(B)$ $\rightarrow H^{1}(X)$. In particular, there is a continuous $f: X \rightarrow A_{X}$ such that $f(x)=0$ and $f^{*}: H^{1}\left(A_{X}\right) \rightarrow H^{1}(X)$ is an isomorphism.

Proof. By Theorem 1.1, there is an $F \in \operatorname{Mor}_{S C}(X, B)$ with $F^{*}=b$. By Theorem 1.1 of [6] there is a continuous $f: X \rightarrow B$ with $f(x)=0$ and $S(f)=F$. Thus $f^{*}=F^{*}=b$. The last part of the theorem follows from Corollary 1.5 and the fact that $H^{1}(X)$ is torsion free and thus that $A_{X}$ is a compact connected abelian topological group. 
1.7. Corollary. Let $X$ be a continuum with $x \in X$ and $B$ be a compact connected abelian topological group. Let $A_{X}=\operatorname{char} H^{1}(X)$. Let $f: X \rightarrow A_{X}$ be a continuous function with $f(x)=0$ and $f^{*}: H^{1}\left(A_{X}\right) \rightarrow H^{1}(X)$ an isomorphism. Then if $g: X \rightarrow B$ is continuous with $g(x)=0$, then there is a continuous homomorphism $\pi: A_{X} \rightarrow B$ such that $\pi \circ f$ is bomotopic to $g$.

Proof: Let $g^{*}: H^{1}(B) \rightarrow H^{1}(X)$ and define $b=f^{*-1} \circ g^{*}$. Then $b: H^{1}(B) \rightarrow$ $H^{1}\left(A_{X}\right)$ induces a homomorphism $\pi: A_{X} \rightarrow B$ such that $\pi^{*}=b$. Then $(\pi \circ f)^{*}=$ $f^{*} \circ \pi^{*}=f^{*} \circ\left(f^{*-1} \circ g^{*}\right)=g^{*}$. Thus $S(\pi \circ f)=S(g)$ by Theorem 1.1. But $\pi \circ$ $f(x)=g(x)=0$ and by Theorem 1.2 of [6], $\pi \circ f$ is homotopic to $g$.

2. Local connectivity and $H^{1}(X)$. In this section of the paper we show that if $X$ is a locally connected continuum, then $H^{1}(X)$ has property $\mathrm{L}$. Property $\mathrm{L}$ arises in the study of compact abelian topological groups $A$ where it is well known [10, Theorem 48, p. 260] that $A$ is locally connected if and only if char $A$ has property L. If $A$ is connected, then this theorem together with Theorem 1.4 of the previous section shows that $A$ is locally connected if and only if $H^{1}(A)$ has property $L$.

2.1. Definition. Let $G$ be an abelian group and $H$ a subgroup of $G$. Then $H$ is said to admit division if whenever $g \in G$ and $n$ is a positive integer, then $n g \in H$ implies that $g \in H$. This is equivalent to saying that $G / H$ is torsion free. The group $G$ is said to have property $L$ if every finite subset of $G$ is contained in a finitely generated subgroup that admits division.

2.2. Theorem. Let $X$ be a continuum. Then if $X$ is locally connected, then $H^{1}(X)$ bas property $\mathrm{L}$.

Before proceeding to the proof of Theorem 2.2 we will make some preliminary remarks to make the proof clearer. We summarize these remarks in four claims for easy reference in the proof of Theorem 2.2. First of all, suppose that $G$ is an abelian group that does not have property $L$. Then there is a finite subset $\left\{g_{1}, \cdots, g_{n}\right\}$ $\subset G$ such that for every finitely generated subgroup $H$ with $\left\{g_{1}, \cdots, g_{n}\right\} \subset H \subset G$, $G / H$ is not torsion free. This implies that if $H=\{g \in G$ : for some integer $n, n g$ is in the subgroup generated by $\left\{g_{1}, \cdots, g_{n}\right\}$, then $H$ cannot be finitely generated since $G / H$ is torsion free. Note that $H$ has finite rank and that $H$ does not have property $L$. That is, if $G$ does not have property $L$, then there is a subgroup $H$ of $G$ such that $H$ admits division in $G, H$ is of finite rank, and $H$ does not have property $L$. Thus we have shown the following.

Claim 1. If $G$ is an abelian group which does not have property $L$, then there is a subgroup $H$ of $G$ which admits division in $G$ such that $H$ is of finite rank and $H$ does not have property $L$.

We now review some properties of Pontryagin duality. If $A$ is a compact abelian topological group, then $\operatorname{dim} A=\operatorname{rank}$ char $A$. Also, if $A$ is a compact abelian 
topological group, then $A$ is metrizable if and only if char $A$ is countable. In particular, this last remark implies that if $H$ is an abelian group which is torsion free and of finite rank, then $A=$ char $H$ must be metrizable since $H$ must be countable under these circumstances.

Claim 2. If $H$ is a torsion free abelian group which is of finite rank, then char $H=A$ is a compact connected abelian topological group with $\operatorname{dim} A=\operatorname{rank} H$ and $A$ metrizable.

Another point to be made has to do with the results in [2]. Let $G$ be a compact connected topological group which is finite-dimensional. Then if $G_{0} \subset G$ is the arc component of the identity $e$ in $G$, then the locally arcwise connected topology on $G_{0}$ makes $G_{0}$ into a Lie group $L$. Now $G_{0}$ is dense in $G$ and thus by [2] we can say that there is a Lie group $L$ and a one-to-one continuous homomorphism $\phi$ : $L \rightarrow G$ such that $\phi(L)$ is precisely the arc component of $e$ in $G$ and is dense in $G$. If $G$ is abelian, then $L$ must obviously also be abelian and thus be of the form $R^{k} \times T^{m}$. The first part of the next claim summarizes this.

Claim 3. Let $A$ be a compact connected abelian topological group which is finite-dimensional. Then there is a one-to-one continuous homomorphism $\phi: R^{k} \times$ $T^{m} \rightarrow A$ such that $\phi\left(R^{k} \times T^{m}\right)$ is precisely the arc component of 0 in $A$ and is dense in $A$. If $Q \subset \phi\left(R^{k} \times T^{m}\right)$ is a peano continuum, then $\phi^{-1} \mid Q$ is continuous.

The last part of Claim 3 comes from [2, Theorem 3.2] and the fact that $R^{k} \times T^{m}$ is just the arc component of 0 in $A$ with the associated locally arcwise connected topology described in [2].

We will need one other result in the proof of Theorem 2.2 which is proved in a claim in the proof of Theorem 1.2 in [6].

Claim 4. Let $A$ be a compact connected abelian topological group and let $T^{m}$ be a subgroup of $A$. Then $A \simeq B \times T^{m}$ for some compact connected abelian topological group $B$.

We also use the fact that $H^{1}(X)$ is torsion free for all $X$.

Proof of Theorem 2.2. Assume that $X$ is a locally connected continuum and that $H^{1}(X)$ does not have property $L$. Then by Claim 1 , there is a subgroup $M$ of $H^{1}(X)$ of finite rank which admits division in $H^{1}(X)$ and does not have property $L$. Let $b: M \rightarrow H^{1}(X)$ be the inclusion homomorphism. Let $B=\operatorname{char} M$ and let $f: X \rightarrow B$ be a continuous map given by Corollary 1.6 such that $f^{*}=b: H^{1}(B) \rightarrow H^{1}(X)$ with $0 \in f(X)$ in $B$. Observe that $H^{1}(X)$ is torsion free and thus $M$ is also torsion free. Thus by Claim 2, $B=\operatorname{char} M$ is metrizable. By Claim 3, there is a one-to-one continuous $\phi: R^{k} \times T^{m} \rightarrow B$ with $\phi\left(R^{k} \times T^{m}\right)$ the arc component of 0 in $B$ and dense in $B$. Since $X$ is locally connected, so is $f(X) \subset B$. Since $B$ is metrizable, so is $f(X)$. Thus $f(X)$ is a peano continuum and thus $f(X) \subset \phi\left(R^{k} \times T^{m}\right)$. By Claim 3, $\phi^{-1} \mid f(X)$ is continuous and thus there is a continuous $g: X \rightarrow R^{k} \times T^{m}$ such that $g \circ \phi=f$. Clearly $g$ is homotopic to a map $g^{\prime}: X \rightarrow\{0\} \times T^{m}$. Now $\phi\left(\{0\} \times T^{m}\right) \simeq T^{m}$ 
is a subgroup of $B$ and thus by Claim $4, B \simeq A \times T^{m}$ for some compact connected abelian topological group $A$. Thus $f$ is homotopic to an $f^{\prime}: X \rightarrow\{0\} \times T^{m} \subset A \times$ $T^{m}=B$. However, this implies that $f^{*}: H^{1}(B) \rightarrow H^{1}(X)$ factors through the group $Z^{m}=H^{1}\left(T^{m}\right), H^{1}(B) \rightarrow Z^{m} \rightarrow H^{1}(X)$. This implies that $M=f^{*}\left(H^{1}(B)\right)$ is a quotient of a subgroup of $Z^{m}$ and thus a free abelian group $Z^{p}$ of rank $p \leq m$. This is a contradiction since $M$ did not have property $L$ and $Z^{p}$ has property $L$. This contradiction shows that if $X$ is locally connected, then $H^{1}(X)$ has property $L$.

Now we show that there are peano continua $X$ for which $H^{n}(X)$ does not have property $L$ for $n>1$ so that Theorem 2.2 does not generalize to the higher-dimensional groups. In the next section, however, we will show that if $X$ is a movable compactum, then $H^{n}(X) /$ Tor $H^{n}(X)$ has property $L$ for every $n$.

Let $X$ be a metric compactum and $D$ be the standard Cantor set in the unit interval $I=[0,1]$. Then there is a continuous function $f(D)=X$ and we can form the space $X_{f}=I U_{f} X$. Then $X_{f}$ is a peano continuum containing $X$ as a closed subspace. One can think of $X$ as being obtained from $X$ by adjoining countably many arcs to $X$ with their endpoints attached to points of $X$ and with their diameters going to zero. Let $f^{\prime}: I \rightarrow X$ be the map of $I$ onto $X$ induced by $f$ and let $I-D$ $=\bigcup_{i=1}^{\infty} O_{i}$ where $\left\{O_{i}\right\}$ is a disjoint collection of open arcs in $I$. If $V$ is an open set in $X$, containing $X$, then there are a finite number of the $O_{i}$ 's, $O_{n_{1}}, \cdots, O_{n_{k}}$, such that $f^{\prime}\left(O_{n_{i}}\right) \not \subset V$. This is because $f^{\prime-1}(V)$ is an open set in $I$ containing $D$.

2.3. Theorem. Let $X$ be a metric continuum and $n>1$. Then $H^{n}(X) \simeq H^{n}\left(X_{f}\right)$.

Proof.By Theorem 1.2 we may assume that $H^{n}\left(X_{f}\right)=\operatorname{Mor}_{H C}\left(X_{f}, K(Z, n)\right)$ and $H^{n}(X)=\operatorname{Mor}_{H C}(X, K(Z, n))$. Since $Z$ is countable, $K(Z, n)$ has the homotopy type of a locally finite connected polyhedron and thus has the homotopy type of an ANR. Thus we may assume that it is an ANR. Define $b: \operatorname{Mor}_{H C}\left(X_{f}, K(Z, n)\right) \rightarrow$ $\operatorname{Mor}_{H C}(X, K(Z, n))$ by $b(H(f))=H(f \mid X)$. Certainly $b$ is well defined and a homomorphism. We will now show that it is one-to-one and onto.

Suppose that $b\left(H\left(f_{1}\right)\right)=b\left(H\left(f_{2}\right)\right)$. We will show that this implies that $H\left(f_{1}\right)=$ $H\left(f_{2}\right)$ and thus that $b$ is one-to-one. By the definition of $b, f_{1} \mid X$ is homotopic to $f_{2} \mid X$. Let $H: X \times I \rightarrow K(Z, n)$ be a homotopy from $f_{1} \mid X$ to $f_{2} \mid X$. Let $A \subset X_{f} \times I$ be the subset consisting of $X \times I \cup X_{f} \times\{0\} \cup X_{f} \times\{1\}$ and let $H^{\prime}: A \rightarrow K(Z, n)$ be defined by $H^{\prime}\left|X \times I=H, H^{\prime}\right| X_{f} \times\{0\}=f_{1}$ and $H^{\prime} \mid X, \times\{1\}=f_{2}$. Then $H^{\prime}$ is well defined and continuous. Since, $K(Z, n)$ is an ANR, there is an extension of $H^{\prime}$ to $H^{\prime \prime}: U \rightarrow K(Z, n)$ where $U$ is an open set in $X_{f} \times I$ containing $A$. Now $U$ contains an open set of the form $V \times I$ where $V$ is open in $X$, and contains $X$. Let $O_{n_{1}}$, $\cdots, O_{n_{k}}$ be the open complementary arcs in $I-D$ such that $f^{\prime}\left(O_{n_{i}}\right) \not \subset V$. Let $I_{1}$, $\cdots, I_{k}$ be closed arcs in $O_{n_{1}}, \cdots, O_{n_{k}}$, respectively, such that $f^{\prime}\left(I-\bigcup_{i=1}^{k} I_{i}\right) \subset V$. Let $V^{\prime}=V-f\left(U_{i=1}^{k} I_{i}\right)$. Let $a_{i}<b_{i}$ be the endpoints of $I_{i}$. Then the complement 
of $V^{\prime} \times I$ in $X_{f} \times I$ is just $\bigcup_{i=1}^{k} f^{\prime}\left(I_{i}\right) \times I$ which is a finite collection of disjoint squares $I^{2}$ and the boundary of $V^{\prime} \times I$ in $X, \times I$ is just $\bigcup_{i=1}^{k}\left\{a_{i}, b_{i}\right\} \times I$. Consider $H^{\prime \prime} \mid\left[\overline{V^{\prime} \times I} \cup X_{f} \times\{0,1\}\right]=H^{\prime \prime \prime}$. We will now extend $H^{\prime \prime \prime}$ to all of $X_{f} \times I$. Now $H^{\prime \prime \prime}$ is defined on the boundary of $f^{\prime}\left(I_{i}\right) \times I$ which is homeomorphic to a circle. Since $n>1, K(Z, n)$ is simply connected and thus $H^{\prime \prime \prime} \mid \operatorname{Bd}\left(f^{\prime}\left(I_{i}\right) \times I\right)$ can be extended to all of $f^{\prime}\left(I_{i}\right) \times I$ for each $i$. This gives the extension of $H^{\prime \prime \prime}$ to an $\tilde{H}: X, \times I \rightarrow$ $K(Z, n)$. Then $\widetilde{H}$ is a homotopy of $f_{1}$ to $f_{2}$ and $H\left(f_{1}\right)=H\left(f_{2}\right)$. Thus we have shown $b$ to be one-to-one.

To show that $b$ is onto let $H(g) \in \operatorname{Mor}_{H C}(X, K(Z, n))$. Then there is an extension $g^{\prime}$ of $g$ to a neighborhood $V$ of $X$ in $X_{f}$. Let $I_{1}, \cdots, l_{k}$ be a finite number of closed arcs in $I-D$ having property that $f^{\prime}\left(I-\bigcup_{i=1}^{k} I_{i}\right) \subset V$. Let $a_{i}<b_{i}$ be the endpoints of $I_{i}$ in $I$. Let $V^{\prime}=X_{f}-\bigcup_{i=1}^{k} f^{\prime}\left(I_{i}\right)$. Then the extension $g^{\prime}$ of $g$ given above is onto $\bar{V}^{\prime}$. Then extend $g^{\prime} \mid \bar{V}^{\prime}$ along the arcs $f^{\prime}\left(I_{i}\right)$ in any manner using the fact that $K(Z, n)$ is arcwise connected. This gives an extension $g^{\prime \prime}$ of $g$ to all of $X_{f}$. Then $b\left(H\left(g^{\prime \prime}\right)\right)=H\left(g^{\prime \prime} \mid X\right)=H(g)$ and $b$ is onto. Theorem 2.3 is now proved.

2.4. Corollary. Let $H$ be an arbitrary countable abelian group which is torsion free and $n>1$. Then there is a peano continuum $X$ such that $H^{n}(X) \simeq H$.

Proof. Let $A=$ char $H$. Then $A$ is a compact connected abelian topological group with $H^{1}(A) \simeq H$. Note that $A$ is metrizable since the weight of $A$ is countable, $w(A)=$ card $H$. Let $B=\Sigma^{n-1} A$, the $(n-1)$-fold suspension of $A$. Then $H^{n}(B) \simeq H$. Let $X=B_{f}$. Then $H^{n}(X) \simeq H$ with $X$ a peano continuum.

This construction will be used in a later section of the paper to construct nonmovable peano continua.

3. Čech cohomology and topological groups. Let $G$ be a compact connected topological group. We show here as an easy consequence of the results in $\$ 1$ that $H^{1}(G)$ is determined by the commutator subgroup of $G$ in a manner made precise by the next theorem.

3.1. Theorem. Let $G$ be a compact connected topological group and let $D$ be its commutator subgroup. Then $H^{1}(G) \simeq H^{1}(G / D)$.

Proof. Let $A_{G}=\operatorname{char} H^{1}(G)$ and let $f: G \rightarrow A_{G}$ be continuous with $f(e)=0$ such that $f^{*}: H^{1}\left(A_{G}\right) \rightarrow H^{1}(G)$ is an isomorphism by Corollary 1.5. By Theorem 1.3, $f$ may be taken to be a continuous homomorphism. Note that $D \subset \operatorname{ker} f$. Now $G / D=B$ is a compact connected abelian topological group and $g: G \rightarrow B$ is continuous. Thus by Corollary 1.7 there is a continuous homomorphism $\pi: A_{G} \rightarrow B$ such that $\pi \circ f$ is homotopic to $g$. But by Theorem 1.3, $\pi \circ f=g$. Thus $D=$ $\operatorname{ker} g \supset \operatorname{ker} f$ and $D=\operatorname{ker} f$. Note that since $f^{*}$ is an isomorphism, $f$ must be onto, 
otherwise $f(G)$ would be a proper closed subgroup of $A_{G}$ and $f^{*}$ could not be an isomorphism. Thus $A_{G} \simeq G / \operatorname{ker} f=G / D=B$.

3.2. Remark. In this case the map $f: G \rightarrow A_{G}$ which induces an isomorphism $f^{*}: H^{1}\left(A_{G}\right) \rightarrow H^{1}(G)$ is onto. However, if $X$ is a continuum, then the continuous map given in Corollary 1.6 may not be onto even in the case that $f: X \rightarrow A_{X}$ with $f^{*}: H^{1}\left(A_{X}\right) \rightarrow H^{1}(X)$ an isomorphism. For example let $X=T \vee T$ where $T$ is the circle. Then $H^{1}(X) \simeq Z^{2}$ and we could let $f: X \rightarrow T^{2}=$ char $H^{1}(X)$ map $X$ onto two generating circles in $T^{2}$. Since $f(X)$ may not be all of $A_{X}$, one can see the necessity of using the techniques in the proof of Theorem 2.2.

4. Movability of compacta. In this section we show that if $X$ is movable, then $H^{n}(X) /$ Tor $H^{n}(X)$ has property $\mathrm{L}$. This allows us to construct a variety of nonmovable peano continua. For convenience of notation in this section if $G$ is an abelian group, then we let $\hat{G}=G /$ Tor $G$. If $b: G \rightarrow H$ is a homomorphism, then $\hat{b}: \hat{G} \rightarrow \hat{H}$ is the naturally induced homomorphism.

4.1. Lemma. Let $G$ be an abelian group which is the direct limit of the direct system of abelian groups $\left\{G_{a} ; \pi_{a \beta} ; \alpha \leq \beta \in A\right\}$. Then $\hat{G}$ is the direct limit of the direct system $\left\{\hat{G}_{\alpha} ; \hat{\pi}_{\alpha \beta} ; a \leq \beta \in A\right\}$.

Proof. Let the homomorphisms $\pi_{a}: G \rightarrow G_{a}$ make $G$ the direct limit of the direct system $\left\{G_{a} ; \pi_{a \beta} ; \alpha \leq \beta \in A\right\}$ and let $\hat{\pi}_{a}: \hat{G}_{a} \rightarrow \hat{G}$ be the naturally induced homomorphisms for $a \in A$. We will show that the homomorphisms $\left\{\hat{\pi}_{a}\right\}$ make $\hat{G}$ the direct limit of the direct system $\left\{\hat{G}_{a}\right\}$. Clearly $\hat{\pi}_{\beta} \circ \hat{\pi}_{\alpha \beta}=\hat{\pi}_{\alpha}$ for all $\alpha \leq \beta \in A$. Also if $[b] \in \hat{G}$, then there is an $a \in A$ and $b_{a} \in G_{a}$ such that $\pi_{a}\left(b_{a}\right)=b$. Thus $\hat{\pi}_{a}\left(\left[b_{a}\right]\right)=[b]$. Thus $\bigcup_{a \in A} \hat{\pi}_{a}\left(G_{a}\right)=\hat{G}$. All that needs to be shown is that if $\hat{\pi}_{\alpha}\left(\left[b_{a}\right]\right)=\hat{\pi}_{\beta}\left(\left[b_{\beta}\right]\right)$, then there is a $\gamma \geq a, \beta$ such that $\hat{\pi}_{a \gamma}\left(\left[b_{a}\right]\right)=\hat{\pi}_{\beta \gamma}\left(\left[b_{\beta}\right]\right)$. To show this, note that $d=\pi_{a}\left(b_{a}\right)-\pi_{\beta}\left(b_{\beta}\right) \in$ Tor $G$. Let $\delta \geq \alpha, \beta$ and $c=\pi_{a \delta}\left(b_{\alpha}\right)-$ $\pi_{\beta \delta}\left(b_{\beta}\right)$. Then $\pi_{\delta}(c)=d$. Since $d \in$ Tor $G$, there is an $n \geq 1$ such that $n d=0$. Then there is a $\gamma \geq \delta$ such that $\pi_{\delta \gamma}(n c)=n \pi_{\delta \gamma}(c)=0$. But then $\pi_{a \gamma}\left(b_{\alpha}\right)-$ $\pi_{\beta \gamma}\left(b_{\beta}\right)=\pi_{\delta \gamma}\left(\pi_{a \delta}\left(b_{\alpha}\right)-\pi_{\beta \delta}\left(b_{\beta}\right)\right)=\pi_{\delta \gamma}(c) \in$ Tor $G$. Thus $\hat{\pi}_{a \gamma}\left(\left[b_{\alpha}\right]\right)=\hat{\pi}_{\beta \gamma}\left(\left[b_{\beta}\right]\right)$ for this $\gamma \in A$.

\subsection{Lemma. Let $X$ be a compact space and $X$ be the inverse limit of the} inverse system $\left\{X_{\alpha} ; \pi_{a \beta} ; \alpha \leq \beta \in A\right\}$. Then $\hat{H}^{n}(X)$ is the direct limit of the direct system $\left\{\hat{H}^{n}(X) ; \hat{\pi}_{a \beta}^{*} ; \alpha \leq \beta \in A\right\}$.

Proof. By the continuity of Čech cohomology, $H^{n}(X)$ is the direct limit of the direct system $\left\{H^{n}\left(X_{\alpha}\right) ; \pi_{\alpha \beta}^{*} ; \alpha \leq \beta \in A\right\}$. By Lemma 4.1, $\hat{H}^{n}(X)$ is the direct limit of the direct system $\left.\hat{H}^{n}\left(X_{\alpha}\right) ; \hat{\pi}_{\alpha \beta}^{*} ; a \leq \beta \in A\right\}$.

Now we recall the definition of movability as given in [7] and then state and prove the main theorem of this section. We assume familiarity with the ANR-sys- 
tem approach to shape in [8] and the equivalence of the approaches in [3] and [8] as shown in [5].

4.3. Definition. An ANR-system $X=\left\{X_{a} ; \pi_{a \beta} ; \alpha \leq \beta \in A\right\}$ is said to be movable provided that for each $a \in A$, there is a $\beta \geq \alpha$ such that for every $\gamma \geq \beta$ there is a map $r^{\beta \gamma}: X_{\beta} \rightarrow X_{y}$ such that $H\left(\pi_{a \gamma}{ }^{\circ}{ }^{\beta \gamma}\right)=H\left(\pi_{a \beta}\right)$. A compactum $X$ is said to be movable if an ANR-system associated with $X$ is movable. As is shown in [7], if one ANR-system associated with $X$ is movable, then every one is and movability is a shape invariant.

4.4. Theorem. If $X$ is a movable compactum, then $\hat{H}^{n}(X)$ bas property $\mathrm{L}$ for every $n \geq 0$.

Proof. Let $X=\left\{X_{a} ; \pi_{a \beta} ; a \leq \beta \in A\right\}$ be an ANR-system associated with $X$ with each $X_{a}$ a finite polyhedron. Let $\pi_{a}: X \rightarrow X_{a}$ be the projection maps making $X$ the inverse limit of the system $X$. Note that $H^{n}\left(X_{\alpha}\right)$ and $\hat{H}^{n}\left(X_{\alpha}\right)$ are finitely generated for all $\alpha \in A$. By Lemma 4.2, $\hat{H}^{n}(X)$ is the direct limit of the direct system $\left\{\hat{H}^{n}\left(X_{\alpha}\right) ; \hat{\pi}_{a \beta}^{*} ; a \leq \beta \in A\right\}$. Now we will show that $\hat{H}^{n}(X)$ has property $L$ if $X$ is movable. Let $\left\{a_{1}, \cdots, a_{k}\right\}$ be an arbitrary finite subset of $\hat{H}^{n}(X)$. There is an $a \in A$ such that $\hat{\pi}_{a}^{*}\left(\hat{H}^{n}\left(X_{\alpha}\right)\right)$ contains $\left\{a_{1}, \cdots, a_{k}\right\}$. Since $X$ is movable, let $\beta \geq a$ be such that for $\gamma \geq \beta$ there is an $r^{\beta \gamma}: X_{\beta} \rightarrow X_{\gamma}$ such that $H\left(\pi_{a \gamma} \circ{ }_{r} \beta \gamma\right)=$ $H\left(\pi_{\alpha \beta}\right)$. Now let $L$ be the subgroup of $\hat{H}^{n}(X)$ defined by $g \in L$ if and only if $m g \epsilon$ $\hat{\pi}_{\alpha}^{*}\left(\hat{H}^{n}\left(X_{\alpha}\right)\right)$ for some positive integer $m$. We will now show that $L \subset \hat{\pi}_{\beta}^{*}\left(\hat{H}^{n}\left(X_{\beta}\right)\right)$ and thus that $L$ is finitely generated. By the definition of $L, L$ will admit division in $\hat{H}^{n}(X)$ and thus $\hat{H}^{n}(X)$ will have property $L$.

Let $m g \in \hat{\pi}_{a}^{*}\left(\hat{H}^{n}\left(X_{\alpha}\right)\right)$ for some $m \geq 1$. Then there is a $\delta \geq \beta$ such that $g \epsilon$ $\hat{\pi}_{\delta}^{*}\left(\hat{H}^{n}\left(X_{\delta}\right)\right)$. Let $e \in \hat{H}^{n}\left(X_{a}\right)$ be such that $\hat{\pi}_{a}^{*}(e)=m g$ and let $f \in \hat{H}^{n}\left(X_{\delta}\right)$ be such that $\hat{\pi}_{\delta}^{*}(f)=g$. Then $\hat{\pi}_{a}^{*}(e)=\hat{\pi}_{\delta}^{*}(m f)=m g$. Thus there is a $\gamma \geq \delta$ such that $\hat{\pi}_{\alpha \gamma}^{*}(e)=\hat{\pi}_{\delta \gamma}^{*}(m f)$. Let $r^{\beta \gamma}: X_{\beta} \rightarrow X_{\gamma}$ be as in the definition of movability and let $b=\left({ }_{r} \beta \gamma_{*}\right)$. Then $b \circ \hat{\pi}_{a \gamma}^{*}=\hat{\pi}_{a \beta}^{*}$. Now $b(m f)=m b(f)$ and thus $b(f)$ is an $m$ th root of $b(m f)$ in $\hat{H}^{n}\left(X_{\beta}\right)$. However, $\hat{\pi}_{a \beta}^{*}(e)=b \circ \hat{\pi}_{a y}^{*}(e)=b(m f)$. Thus $b(f)$ is an $m$ th root of $\hat{\pi}_{\alpha \beta}^{*}(e)$ in $\hat{H}^{n}\left(X_{\beta}\right)$. Now $m \hat{\pi}_{\beta}^{*}(b(f))=\hat{\pi}_{\beta}^{*}(m b(f))=\hat{\pi}_{\beta}^{*}\left(\hat{\pi}_{\alpha \beta}^{*}(e)\right)=$ mg. Thus $m \hat{\pi}_{\beta}^{*}(b(f))=m g$. But $\hat{H}^{n}(X)$ is torsion free. Thus $m$ th roots in $\hat{H}^{n}(X)$ are unique. Thus $\hat{\pi}_{\beta}^{*}(b(f))=g$. Thus $g \in \hat{\pi}_{\beta}^{*}\left(\hat{H}^{n}\left(X_{\beta}\right)\right)$ and $L \subset \hat{\pi}_{\beta}^{*}\left(\hat{H}^{n}\left(X_{\beta}\right)\right)$ as asserted and $\hat{H}^{n}(X)$ has property $L$.

4.5. Corollary. If $X$ is movable and $H^{n}(X)$ is torsion free, then $H^{n}(X)$ bas property $\mathbf{L}$.

It may have occurred to the reader to question whether Theorem $4.4 \mathrm{might}$ not be true for $H^{n}(X)$ rather than $\hat{H}^{n}(X)$ in the statement of the theorem. The following example shows that this is not the case. 
4.6. Example. It is well known that $H^{2}\left(R P^{3}\right)=Z_{2}$ where $R P^{3}$ is a 3-dimensional real projective space. Let $X_{n}=\bigvee_{i=1}^{n} R P^{3}$ and for $n \leq m$, define $\pi_{n m}: X_{m} \rightarrow X_{n}$ to be the map which collapses $\bigvee_{i=n+1}^{m} R P^{3}$ to a point and is the identity on $\bigvee_{i=1}^{n} R P^{3}$. Let $X$ be the inverse limit of $\left\{X_{n}\right\}$. Then $X$ is a 3-dimensional metric continuum which is clearly movable. However, $H^{2}(X)=\bigoplus_{i=1}^{\infty} Z_{2}$ and this group does not have property $L$.

We are now able to construct a variety of nonmovable peano continua using these results and the construction described in $\$ 2$.

4.7. Theorem. For every positive integer $n>1$, there are $2^{x_{0}}$ nonbomeomorphic n-dimensional peano continua which are nonmovable.

Proof. There are $2^{x_{0}}$ torsion free abelian groups of rank $1\left\{H_{a}: a \in A\right\}$ no two of which are isomorphic and none of which have property L. Let $\Sigma_{a}=$ char $H_{a}$ and then let $X_{a}=\Sigma^{n-1} \Sigma_{a}$ be the $(n-1)$-fold suspension of $\Sigma_{a}$. Then $X_{a}$ is a metric continuum and $H^{n}\left(X_{a}\right)=\hat{H}^{n}\left(X_{a}\right)=H_{a}$. Clearly $X_{a}$ is $n$-dimensional. Let $Z_{a}$ be the peano continuum $\left(X_{a}\right)$, as described in $\$ 2$. Then $H^{n}\left(Z_{a}\right)=H_{a}$. Clearly $\operatorname{dim} Z_{a}=n$ and in fact $S d\left(Z_{\alpha}\right)=n$ since $H^{n}\left(Z_{a}\right) \neq 0$. Since $\hat{H}^{n}\left(Z_{a}\right)$ does not have property $L, Z_{a}$ cannot be movable. If $Z_{\alpha}$ were homeomorphic to $Z_{\beta}$, then $H_{\alpha}$ would be isomorphic to $H_{\beta}$ which would imply that $a=\beta$. Thus $\left\{z_{\alpha}: \alpha \in A\right\}$ is the desired collection of peano continua.

In [1] Borsuk constructed a nonmovable peano continuum which was two-dimensional. Note that the construction given in Theorem 4.7 will fail if $n=1$, since a one-dimensional peano continuum is movable. In [9] Overton and Segal give a short proof of a theorem of Mardesic that an $n$-dimensional $L C^{n-1}$ peano continuum is movable. That theorem gives us the following corollary to Theorem 4.4.

4.8. Corollary. Let $X$ be an n-dimensional peano continuum. Then if $X$ is $L C^{n-1}$, then $\hat{H}^{k}(X)$ bas property $\mathrm{L}$ for all $k$.

\section{REFERENCES}

1. K. Borsuk, On a locally connected non-movable continuum, Bull. Acad. Polon. Sci. Sér. Sci. Math. Astronom. Phys. 17 (1969), 425-430. MR 41 \#1012.

2. A. M. Gleason and R. S. Palais, On a class of transformation groups, Amer. J. Math. 79 (1957), 631-648. MR 19, 663.

3. W. Holsztyński, An extension and axiomatic characterization of Borsuk's theory of shape, Fund. Math. 70 (1971), no. 2, 157-168. MR 43 \#8080.

4. P. J. Huber, Homotopical cohomology and Čech cohomology, Math. Ann. 144 (1961), 73-76. MR 24 \#A3646.

5. J. Keesling, Shape theory and compact connected abelian topological groups, Trans. Amer. Math. Soc. (to appear).

6. - Continuous functions induced by shape morphisms, Proc. Amer. Math.

Soc. 41 (1973), 315-320. 
7. S. Mardesič and J. Segal, Movable compacta and ANR-systems, Bull. Polon. Acad. Sci. Sér. Sci. Math. Astronom. Phys. 18 (1970), 649-654. MR 44 \#1026.

8. - Shapes of compacta and ANR-systems, Fund. Math. 72 (1971), 41-59.

MR $45 \# 7686$.

9. R. Overton and J. Segal, A new construction of movable compacta, Glasnik Mat. 6 (26) (1971), 361-363.

10. L. S. Pontrjagin, Continuous groups, 2nd ed., GITTL, Moscow, 1954; English transl., Topological groups, Gordon and Breach, New York, 1966. MR 17, 171; 34 \#1439.

11. W. Scheffer, Maps between topological groups that are homotopic to homomorphisms, Proc. Amer, Math. Soc. 33 (1972), 562-567. MR 46 \#288.

12. E. Spanier, Algebraic topology, McGraw-Hill, New York, 1966. MR 35 \#1007.

13. N. Steenrod, Universal homology groups, Amer. J. Math. 58 (1936), 661-701.

DEPARTMENT OF MATHEMATICS, UNIVERSITY OF FLORIDA, GAINESVILLE, FLORIDA 32601 